
$\$$ Research Square
Preprints are preliminary reports that have not undergone peer review.
They should not be considered conclusive, used to inform clinical practice, or referenced by the media as validated information.

\title{
Sex differences in the association between self-reported sleep duration, insomnia symptoms and cardiometabolic risk factors: cross-sectional findings from ELSA-Brasil
}

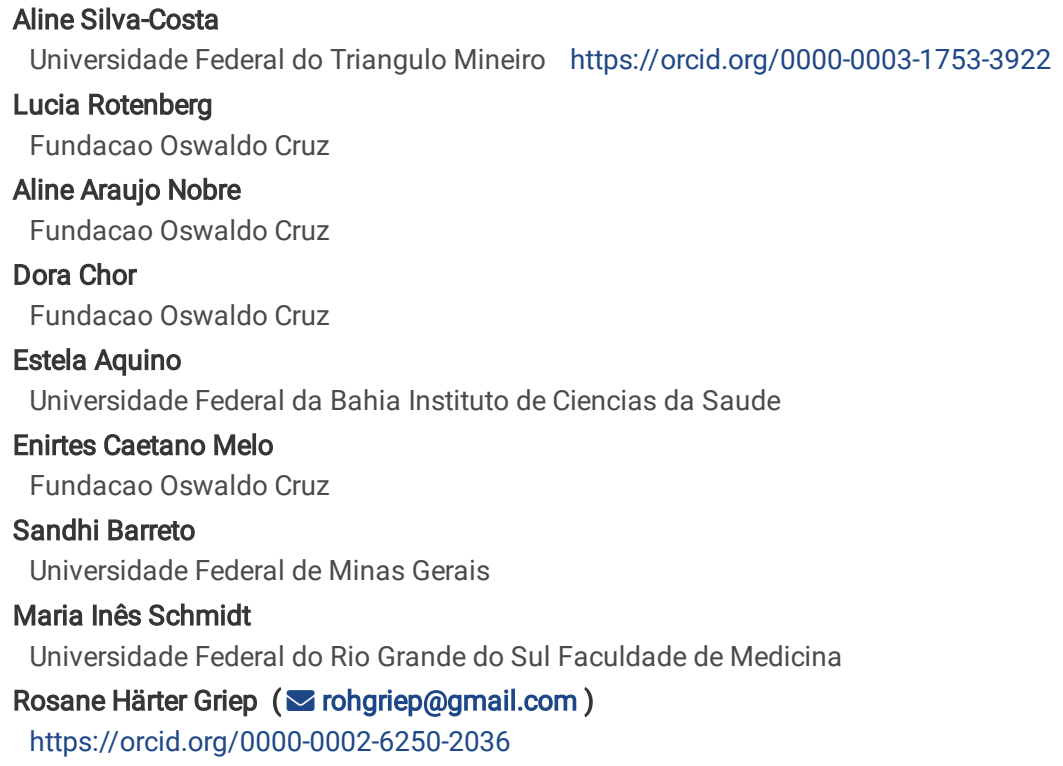

Research

Keywords: sleep duration, insomnia, hypertension, obesity and glycated hemoglobin

Posted Date: December 16th, 2019

DOI: https://doi.org/10.21203/rs.2.18615/v1

License: (c) (7) This work is licensed under a Creative Commons Attribution 4.0 International License. Read Full License 


\section{Abstract}

Background: The U-shaped associations between sleep durations and cardiometabolic risk factors (glycated hemoglobin levels, obesity, hypertriglyceridemia, hypertension and HDL-Cholesterol levels) are still inconclusive. Moreover, as sleep is comprised of quantitative and qualitative aspects, exploring both insomnia symptoms and sleep duration are relevant when evaluating the potential effects of sleep problems on health. The aim was to evaluate sex-specific associations between sleep problems and cardiometabolic risk factors.

Methods: This cross-sectional study used data from wave two of the Brazilian Longitudinal Study of Adult Health (ELSA-Brasil), including 7,491 women and 6,232 men. Questionnaires were administered to provide information about socioeconomic conditions, lifestyle, and sleep characteristics. A 12-hour fasting blood sample was drawn to measure serum cholesterol, triglycerides, and glycated hemoglobin. Blood pressure, weight and height were also measured using standard equipment. Generalized additive models were used to evaluate the curve shape of the relationship between self-reported sleep duration and the outcomes. Logistic regression was performed to investigate the magnitude of the associations of self-reported sleep duration, insomnia symptoms, and short sleep plus insomnia symptoms with cardiometabolic risk factors.

Results: For women, self-reported sleep duration and insomnia symptoms (either separately or linked to short sleep duration) were associated with obesity, hypertension and glycated hemoglobin after adjusting for the confounders. The magnitudes of the associations between self-reported short sleep duration plus insomnia symptoms and the outcomes were slightly increased, considering sleep duration or insomnia symptoms separately. For men, both long sleep duration and insomnia symptoms were associated with hypertriglyceridemia after adjusted for the confounders.

Conclusion: These findings suggest possible sex-specific patterns, since obesity, hypertension and high glycated hemoglobin were associated with selfreported sleep duration and insomnia symptoms in women, but not in men, and reinforce the importance of considering quantitative and qualitative aspects of sleep for the prevention and management of the outcomes.

\section{Background}

Studies into sleep have increased in recent decades, since it was recognized as a public health issue due to the possible negative effects of sleep problems on health. Previous studies have suggested a link between sleep duration and diabetes, obesity, hypertension and metabolic syndrome. ${ }^{1-9}$ Findings related to sleep duration and plasma lipids are scarce and less consistent, mainly because of few studies on this topic. ${ }^{10-12}$ Although some studies have found that both short and long sleep durations are associated with diabetes, ${ }^{5,7,11-12}$ hypertension and obesity, ${ }^{11-12}$ other studies did not observe this U-shaped relationship. ${ }^{3,12-}$

${ }^{16}$ Moreover, the sleep duration range that corresponds to the lowest risk of cardiometabolic diseases remains unclear. In fact, the wide variation in the reference category (i.e, adequate sleep) across studies (from $>6$ hours to $<9$ hours) ${ }^{3,5-6,17}$ clearly shows that the definition of the reference category deserves attention.

Concerning insomnia symptoms (difficulty initiating sleep, difficulty maintaining sleep, early morning awakening), the studies related to cardiometabolic issues are less extensive ${ }^{3}$, compared to the ones related to sleep duration. Having difficulty falling or staying asleep ('all of the time' or 'most of the time') was associated with type 2 diabetes in young female registered nurses, ${ }^{18}$ and with both hypertension and dyslipidemia among public sector employees. ${ }^{19}$ Metaanalysis studies showed that the risk of type 2 diabetes $^{7}$ and hypertension ${ }^{3}$ were, respectively, $84 \%$ and $20 \%$ higher for subjects who reported difficulties in maintaining their sleep. ${ }^{3,7}$ In a meta-analysis including studies on insomnia and obesity published in the past 10 years, the authors stated that associations are weak, particularly for European samples. ${ }^{19}$ These findings stress that there is no single pattern of results and reinforce the need for studies with other population groups.

As sleep is comprised of quantitative and qualitative aspects, exploring both insomnia symptoms and sleep duration are relevant when evaluating the potential effects of sleep problems on health. In this context, the interaction between short sleep duration and insomnia symptoms, which leads to increased health risks has also been discussed. ${ }^{20-21}$ Cai et al ${ }^{21}$ found a significant association between insomnia symptoms and obesity among participants with short sleep duration, but not among those who reported normal sleep duration (6-8 hours). Similarly, short sleep duration with insomnia symptoms was associated with hypertension. ${ }^{23}$ In relation to diabetes, an association was observed in those with chronic insomnia plus short sleep duration, but not among those with insomnia symptoms plus short sleep duration. ${ }^{24}$

The above-mentioned associations do not specifically comment on the possible different effects between men and women, despite the known gender and sex differences in relation to sleep problems. ${ }^{25-27}$ Few studies have investigated sex-specific associations between sleep and negative outcomes related to noncommunicable chronic diseases. ${ }^{5}$ Gender differences have been detected in studies of sleep problems, with significant associations in women (but not men) with regard to hypertension, ${ }^{28}$ the prevalence of dyslipidemia, ${ }^{29}$ and obesity. ${ }^{30}$ Short sleep duration was associated with a greater BMI and waist circumference among women but not among men. ${ }^{31}$ However, as pointed out by Theorell-Haglöw et al. ${ }^{32}$ research on gender differences in sleep medicine appears to be limited. In a meta-analysis study on sleep and type 2 diabetes, Shan et al. ${ }^{5}$ emphasize the relevance of data on sex, which were usually limited in the original studies. These data reinforce the need for testing the hypothesis of sex-differences in the association between sleep patterns and cardiometabolic factors.

The mechanisms involved in the relationships between sleep problems and metabolic disorders include reduced glucose tolerance and compromised insulin sensitivity. ${ }^{1}$ Insulin and glucose alterations associated with sleep restriction may be partially mediated by neuroendocrine changes, including high evening cortisol levels and increased sympathetic nervous system activity and catecholamine production. ${ }^{33}$ Nocturnal sleep deprivation can lead to abnormal cortisol release during the night, which may result in decreased sensitivity to insulin in the morning. Elevations in cortisol and increased sympathetic nervous system 
activity are also related to impaired vasodilatation and hypertension. Short sleep duration also reduces heart rate variability, ${ }^{33}$ which highlights the importance of sleep in the regulation of many cardiometabolic functions ${ }^{1}$.

Given all the gaps related to the associations of sleep duration and insomnia symptoms with cardiometabolic diseases, the present study aims to evaluate sex-specific associations between sleep (self-reported short and long sleep durations and insomnia symptoms) and cardiometabolic risk factors (high glycated hemoglobin levels, obesity, hypertriglyceridemia, hypertension and low high-density lipoprotein [HDL] levels) using data from the Brazilian Longitudinal Study of Adult Health (ELSA-Brasil). Considering that hypertension, obesity, hyperglycemia and dyslipidemia can lead to cardiovascular disease, the leading non-communicable diseases in terms of premature deaths, ${ }^{34}$ this investigation may provide relevant evidence concerning public health priorities. Therefore the objective of this study was to investigate whether there are sex-specific patterns in the association of cardiometabolic risk factors with (i) selfreported short sleep duration, (ii) self-reported long sleep duration, (iii) insomnia symptoms, and (iv) the combination of insomnia symptoms and selfreported short sleep duration.

\section{Methods}

\section{Study population and setting of the study}

This cross-sectional study used data from wave two of the ELSA-Brasil, which was a multicenter study following civil servants aged 35 to 74 yrs old who were recruited between August 2008 and December 2010 (first wave) at public institutions in six Brazilian state capitals (Salvador, Belo Horizonte, Rio de Janeiro, São Paulo, Vitória and Porto Alegre). The second wave re-examined and interviewed 14,014 individuals. The current analyses included 13722 participants (7491 women and 6231 men) with complete data for all variables. A total of 292 participants with missing data on variables related to self-reported sleep duration $(n=98)$, insomnia symptoms $(n=97)$ and outcomes $(n=42)$ was excluded. Participants who reported sleep durations $<3 \mathrm{~h}$ or $>12 \mathrm{~h}$ were also not considered in the analyses ( $n=29$ women and 26 men).

\section{Variables definition}

A comprehensive set of questionnaires was administered to provide detailed information on socioeconomic conditions, habits, lifestyle, and health. The study design, sampling procedures, construction of the questionnaire and quality assurance and control measures were previously detailed. ${ }^{35-36}$

\section{Sleep variables}

- Self-reported sleep duration: The self-report questionnaire included the question "How many hours of sleep do you get in a usual night's sleep? I_|_| hours I___|minutes"14,21,28 Participants were classified into three groups according to the exact sleep duration they reported: the short sleep duration group ( $\leq 6$ hours), adequate sleep duration group ( $>6$ hours and $\leq 8$ hours) and long sleep duration ( $>8$ hours).

- Insomnia symptoms: The following questions were asked: "In relation to your sleep during the night, at home, during the last 4 weeks, how often did you have difficulty in falling asleep?", "... wake up and have difficulty going to sleep again?", and "... wake up before the desired time and not manage to sleep

again?"21,25 Participants who answered never, rarely or sometimes were classified as no complaint. Participants who answered almost always or always were classified as having a complaint. Participants who reported any of the three described complaints were assigned to the insomnia group.

Self-reported short sleep duration plus insomnia symptoms: this variable considered the combination of self-reported sleep duration and insomnia symptoms. We could not create a "long sleep duration + insomnia" category because few participants (18 men and 45 women) were classified in this group. Therefore, participants with short self-reported sleep duration and insomnia symptoms were compared with participants with adequate sleep durations and without insomnia symptoms.

The reliability of insomnia symptoms and self-reported sleep duration questions was assessed using Kappa statistics and intraclass correlation coefficient (ICC) with their respective $95 \%$ confidence intervals (CI). The ICC for test-retest reliability for sleep duration was good (ICC $0.761 ; 95 \% \mathrm{Cl} 0.685$ to 0.819$)$. There was a substantial agreement for insomnia, and Kappa values were 0.759 (95\% $\mathrm{Cl} 0.651$ to 0.867$)$.

\section{Cardiometabolic variables}

A 12-hour fasting blood sample was drawn by venipuncture in the morning (between 7:00 a.m. and 10:00 a.m.) soon after each subject's arrival at the clinic to measure serum cholesterol, triglycerides, and glycated hemoglobin. ${ }^{35-37}$ Participants with glycated hemoglobin levels $\geq 6.5 \%$ were classified in the high glycated hemoglobin group. Hypertriglyceridemia was defined as triglycerides levels $\geq 150 \mathrm{mg} / \mathrm{dL}$ (milligrams per deciliter). $\mathrm{HDL}$ level $<40 \mathrm{mg} / \mathrm{dL}$ for men and $<50 \mathrm{mg} / \mathrm{dL}$ for women defined the low HDL group. Hypertension was defined as a systolic blood pressure $\geq 140 \mathrm{mmHg}$ or, a diastolic blood pressure $\geq 90$ $\mathrm{mmHg}$, or the use of medication to treat hypertension. Weight and height were also collected using standard equipment and techniques. Obesity was defined as body mass index $(\mathrm{BMI}) \geq 30 \mathrm{~kg} / \mathrm{m}^{2}$.

\section{Sociodemographic and health variables}

The questionnaire also provided information on age, sex, education (fundamental, high school or college), menopausal status and leisure physical activity [PA]; this variable was created considering questions related to the frequency, duration and intensity of PA, categorized as follows: 1) none - no PA or some PA but not meeting the other two categories; 2 ) moderate - $\geq 3$ days of vigorous-intensity PA for at least 20 minutes/day or $\geq 5$ days of moderate-intensity PA and/or walking, in combination or alone, at least 30 minutes/day or $\geq 5$ days of any combination of walking and moderate-or-vigorous-intensity PA achieving a minimum of 600 MET [Metabolic Equivalents]-minutes/week; and 3) high - vigorous-intensity PA on at least 3 days, accumulating a minimum of 1500 MET- 
minutes/week or $\geq 7$ days of any combination of walking and moderate-or-vigorous intensity PA, obtained using the International Physical Activity Questionnaire, (IPAQ long version). ${ }^{38}$ Depression symptoms ascertained using the Clinical Interview Schedule - Revised (CIS- R). ${ }^{39}$

\section{Statistical analyses}

Descriptive analyses were performed to characterize the participants by sex. Given the heterogeneity in creating the range of reference categories for sleep duration, firstly Generalized Additive Models with Bernoulli distribution were used to evaluate the curve shape for the relationship between self-reported sleep duration and the outcomes and to determine the cut-off for hours of sleep duration. The models were fitted considering the potential confounders. After determining the cut-offs for self-reported sleep duration ( $\leq 6$ hours; $>6$ hours - $\leq 8$ hours; $>8$ hours), logistic regression analyses were performed to investigate the magnitude of the associations between self-reported sleep duration, insomnia symptoms and the cardiometabolic risk factors (binary outcomes - high glycated hemoglobin levels, obesity, hypertriglyceridemia, hypertension and low HDL levels). The analyses were adjusted for potential confounders. Model 1 tested a crude association. Then, the analyses were adjusted for age and education (model 2 ) and included physical activity and depression symptoms (model 3). The fourth model was adjusted for self-reported sleep duration or insomnia symptoms to investigate independent effects. The last model included menopausal status. Joint associations of insomnia symptoms and self-reported sleep duration with all outcomes were examined considering participants with adequate sleep duration ( $>6$ hours $-\leq 8$ hours) and absence of insomnia symptoms as the reference group.

All analyses were performed using the software R version 2.15.

\section{Results}

Participants in the ELSA-Brasil included 7491 women and 6231 men in this study. For women and men, the mean age was 55.6 years and 55 years. In relation to sleep patterns, $47.6 \%$ and $49.1 \%$ of women and men, respectively, reported short sleep duration; $4.5 \%$ and $3.2 \%$, respectively, reported long sleep duration; and $27.8 \%$ and $19.3 \%$ of women and men, respectively, reported insomnia symptoms. For both women and men, a higher proportion of individuals with selfreported adequate sleep durations was observed among those with a high level of education, high level of physical activity, and obesity and those without depressive symptoms. The group with insomnia symptoms was more likely to include participants with a low level of education, with no physical activity and with depressive symptoms (Table 1).

\section{[TABLE 1]}

For women, a U-shaped relationship was suggested between self-reported sleep duration and obesity, hypertension and glycated hemoglobin after adjusting for the confounders; adequate self-reported sleep duration ( $>6$ hours - $\leq 8$ hours) was not associated with cardiometabolic risk factors. In contrast, no significant association between self-reported sleep duration and the outcomes was observed for men, except for hypertriglyceridemia, which seems to be related mainly to long sleep duration (Figure 1).

\section{[FIGURE 1]}

For women, in age-adjusted analyses revealed that self-reported sleep duration and insomnia symptoms were significantly associated with obesity, hypertension, low HDL, and high glycated hemoglobin levels compared to the respective reference groups. After adjusting for all potential confounders, the associations remained statistically significant for obesity, hypertension, and high glycated hemoglobin level, and the latter showed the strongest association. Also in women, it should be noted that for obesity, hypertension and high glycated hemoglobin levels, the magnitudes of the associations with the combination of self-reported short sleep duration with insomnia symptoms were higher than those observed for the factors analyzed separately (Table 2). As previously highlighted in Figure 1, these associations were not statistically significant for men, except for hypertriglyceridemia; both long sleep duration and insomnia symptoms were associated with hypertriglyceridemia after adjusted for the confounders (Table 3).

\section{[TABLE 2]}

\section{[TABLE 3]}

\section{Discussion}

Results suggest sex-specific patterns, since obesity, hypertension and high glycated hemoglobin were associated with self-reported sleep duration and insomnia symptoms (either separately or linked to short sleep duration) in women, but not in men. In addition, cardiometabolic risk factors were associated with insomnia symptoms plus short sleep duration only in women. In relation to hypertriglyceridemia, statistically significant associations with insomnia symptoms were observed among both women and men.

Observational studies have shown that the prevalence of insomnia symptoms varies from $10 \%$ to $30 \%, 3,21,25$ and similar to our findings, insomnia symptoms are more frequent among women than men. ${ }^{17,25,30}$ This difference is more evident in self-reported data than objective measures. ${ }^{26}$ Regarding sleep duration, our findings indicated that almost half of the sample reported short sleep duration. This prevalence was higher than those observed in other countries. ${ }^{11,21,31}$ However, for long sleep duration, our findings are in agreement with previous studies. ${ }^{22,31}$

- Sex-specific patterns considering short sleep duration

Some studies reported significant associations between sleep problems and cardiometabolic outcomes for women but not men. Similar to our findings, in the Whitehall cohort, short sleep duration was associated with a higher risk of hypertension only in women. ${ }^{28}$ Also, Grandner et al. ${ }^{40}$ evaluated the association 
between hypertension and sleep duration in a large survey population and observed that short sleep was more closely related to hypertension in women than men. A meta-analysis of the association between sleep duration and hypertension revealed that the results from cross-sectional studies suggested statistically significant associations between short sleep duration and hypertension in women but not in men. ${ }^{8}$

In relation to body weight, Westerlund et al. ${ }^{30}$ observed higher BMI values in women and men with short sleep duration compared with adequate sleep duration (6-8 hours). On the other hand, similar to our findings, short sleep duration was associated with a greater BMI among women but not among men. ${ }^{31}$ A recent meta-analysis ${ }^{6}$ showed an association between short sleep duration and the risk for obesity (lowest risk at 7-8 hours sleep/day), stressing that the associations seem to be more evident in women than men. However, conclusions were not yet possible given the few studies addressing possible gender differences. ${ }^{6}$ The same limitation was pointed out by Shan et al., ${ }^{5}$ who found an association between short sleep duration and an increased risk for type 2 diabetes in a meta-analysis of prospective studies with adult populations. In contrast, our findings on the association between short sleep duration and high glycated hemoglobin were not statistically significant in women or men, although a greater tendency for a positive association was observed among women. Regarding plasma lipids, similar to our results, Kim et al. ${ }^{41}$ did not identify associations between short sleep duration and dyslipidemia in women or men. However, meta-analyses highlight the lack of sufficient evidence to support any conclusion on this issue. ${ }^{10,29}$

- Sex-specific patterns considering long sleep duration

Westerlund et al. ${ }^{30}$ also observed higher BMI values in women with long sleep duration. In contrast, long sleep duration was associated with lower BMI values in men. Kim et al. ${ }^{41}$ observed significant associations of long sleep duration with central obesity, low HDL, and high fasting glucose levels among women but not among men. According to our data, long sleep duration was associated with elevated triglyceride levels only among men, ${ }^{41}$ which deserves further investigations. As mentioned above, recent meta-analyses have addressed the association between sleep duration and cardiometabolic risk factors, but sexdifferences approaches are scarce. A reverse J-shaped relationship was described in a recent meta-analysis, with a borderline association between long sleep duration and the risk of obesity. ${ }^{6}$ A meta-analysis of longitudinal studies that examined the association between sleep duration and diabetes showed that long sleep duration were associated with a significantly increased risk of diabetes compared with 7-8 hours of sleep/day. ${ }^{5}$

Concerning the U-shaped relationship between sleep duration and hypertension, a meta-analysis found that the results from prospective studies showed that neither short nor long sleep duration was statistically associated with the risk of incident hypertension. ${ }^{8}$ However, similar to our findings for women, analyses from cross-sectional studies showed that both short and long sleep durations were associated with prevalent hypertension in adults. ${ }^{8}$ Another meta-analysis of cohort studies showed significant associations between short sleep duration and higher risk of hypertension. ${ }^{3}$ However, the authors found no statistical evidence of any effect of long sleep duration on the risk of hypertension. ${ }^{3}$ A meta-analysis of prospective studies identified that short sleep duration was significantly associated with increased risk of obesity, whereas long sleep duration had no effects on obesity. ${ }^{16} \mathrm{~A}$ systematic review with meta-analyses examining the relationship between short sleep duration and several health outcomes identified significant results for diabetes, hypertension, cardiovascular disease and obesity. ${ }^{11}$ The results from this same research group showed that long sleep duration was significantly associated with diabetes and obesity, but not with increased risk of hypertension. ${ }^{12}$ In summary, the literature is not entirely consistent with respect to the U-shaped associations between sleep duration and metabolic outcomes. Some results from meta-analyses including longitudinal studies have showed statistically significant associations of both short and long sleep duration with diabetes and obesity, ${ }^{5,12}$ while other studies that evaluated hypertension and obesity did not identify any associations. ${ }^{3,16}$ Part of these disagreements could be attributed to methodological issues, such as the instrument used to assess sleep duration and the outcomes (subjective or objective measure), the cut-offs for sleep duration, and the duration of follow-up. ${ }^{3}$

- Sex-specific patterns considering insomnia symptoms

Sex-specific patterns concerning the association between insomnia symptoms and cardiometabolic factors are scarce. Cross-sectional analyses ${ }^{30}$ showed higher BMI values in women with insomnia symptoms, and a similar pattern but less marked among men. In contrast to our findings, insomnia was associated with a hypertriglyceridemia in women, but significant results were not found in men. ${ }^{29}$ Also, difficulty maintaining sleep was associated with an increased overall risk for cardiovascular events in women, but not men ${ }^{42}$.

Regarding data from the general population without discussion of sex-specificities, insomnia symptoms were associated with hypertension medication, ${ }^{44}$ dyslipidemia medication, ${ }^{44}$ and type 2 diabetes. ${ }^{7}$ A meta-analysis of cohort studies showed that insomnia symptoms were significantly associated with a higher risk of hypertension. ${ }^{3}$ However, among individuals with adequate sleep duration, insomnia symptoms were not associated with obesity. ${ }^{22}$

- Joint associations of insomnia and short sleep duration

A recent study found a significant association between insomnia symptoms and obesity among participants with short sleep duration, but not among those who reported adequate sleep duration. ${ }^{22}$ In the same way, short sleep duration with insomnia symptoms was associated with hypertension ${ }^{23}$ and diabetes. ${ }^{24}$ In a cross-sectional study, associations between sleep duration and BMI were not modified by insomnia symptoms. ${ }^{30}$

Our findings on the associations between self-reported sleep duration and each outcome independent of insomnia symptoms (and vice versa) also deserve attention. It has been discussed that insomnia and short sleep duration are distinct aspects of sleep that exhibit intersections. We did not find distinct results after adjusting for insomnia or sleep duration, i.e., the magnitude of the association was similar regardless of the adjustment for the other sleep variable. However, the combination of insomnia symptoms and self-reported short sleep duration appears to intensify the adverse effects of sleep problems separately from the independent associations. ${ }^{20-21}$ Therefore, in line with previous studies, ${ }^{23-24}$ we found that the magnitudes of the associations between self-reported 
short sleep duration plus insomnia symptoms and the outcomes were slightly increased. These findings stress the relevance of considering sleep problems as an additional risk of disease.

- Possible explanations and mechanisms

In line with our findings, Prather et al. ${ }^{27}$ showed that associations between sleep quality and biomarkers were stronger in women than in men. Poorer sleep quality was associated with 5-years increases in Interleukin 6, C-reactive protein, and fibrinogen in women but not in men. Therefore, causal pathways may be different for men and women. Therefore, causal pathways may be different for men and women. A laboratory study examining the effects of sleep deprivation on neural cardiovascular control in men and women found that sleep deprivation decreased muscle sympathetic nerve activity only in men, which in turn exerted a protective function on blood pressure. ${ }^{43}$ Concerning differences in levels of reproductive hormones, it is possible that testosterone, which is higher in men, may attenuate the effects of poor sleep on inflammation. Supporting this hypothesis, lower circulating levels of estradiol contribute to the increased levels of inflammatory activity associated with poor sleep in women around the menopausal period ( 55 years). ${ }^{27}$ The association between sleep problems and greater psychological distress in women but not in men $^{26}$ could also explain these differences. Greater psychological distress in women is generally attributed to a more stressful role for women in Western societies.

Although sex-specific association between sleep and cardiometabolic problems represents an important research area, ${ }^{5-6,32}$ the mechanisms underlying the different patterns are not completely elucidated because few studies have investigated sex differences related to this issue. ${ }^{41}$ Therefore, further studies on sex differences and more mechanism studies are needed to clarify this issue.

In a general way, the biological evidence supporting a putative mechanism between sleep deprivation and cardiometabolic outcomes is derived from disturbances in autonomic function, and inflammatory and hormonal profiles. ${ }^{33}$ For instance, sleep reduction affects energy balance, increases energy intake and reduces energy expenditure. Nocturnal awakening is also associated with altered leptin levels that lead to leptin resistance and result in glucose impairment. Elevated appetite could lead to weight gain and in turn, increases insulin resistance. ${ }^{5,32}$ Possible pathways for the association between long sleep duration and cardiometabolic disease are not clear. Some authors discuss the possibility that long sleepers are previously sick individuals, and for that reason, these associations should be observed in this group. Long sleep is also associated with others sleep disorders, such as obstructive sleep apnea, and poorquality sleep (increased sleep fragmentation and more frequent awakenings), which lead to changes in inflammatory markers that have been shown to be associated with metabolic dysregulation. ${ }^{17,33,45}$ Disagreeing with the hypothesis that morbidity leads to long sleep duration, some studies stress the significant associations of long sleep duration with mortality, diabetes and other diseases, after controlling for other comorbidities. ${ }^{17,45-46}$ It should be noted that in the study of the joint associations of insomnia and sleep duration with diabetes, the adjustment for body mass index did not attenuate associations of long sleep duration without insomnia and diabetes. ${ }^{17}$ In this way, a sensitivity analysis by exclusion of the participants diagnosed with myocardial infarction, stroke, and cancer during the first 2 years of follow-up did not change the association between long sleep duration and diabetes. ${ }^{45} \mathrm{Clearly}$, there is a demand for further studies on long sleepers.

- Strengths and weakness

The strengths of this study are the large multicenter sample of middle-aged Brazilians, the use of standard equipment and techniques and the rigorous quality control of interviews and all measurements. All outcomes included were evaluated by objective measures. Also, we investigated the relationship between sleep duration and the cardiometabolic risk factors, examining firstly if the shape of these possible associations was linear or nonlinear in order to determine the cut off points for sleep duration based on the patterns of our sample.

The limitations of this study include the fact that all sleep variables were self-reported. Also, the insomnia symptoms did not include information related to daytime dysfunction. Although measuring objective sleep duration is more accurate, this procedure is generally not feasible in large epidemiological samples. ${ }^{5,10}$ The current study demonstrated substantial reliability for insomnia symptoms and self-reported sleep duration questions. Also, objective sleepduration measures seemed to be more strongly associated with hypertension than self-reported sleep duration, with no statistically significant differences. ${ }^{3}$ Second, we did not have enough information to distinguish participants who were naturally short sleepers (short sleep duration but feel rested) from the others, and there may be differences between those groups. However, the investigation of individuals who reported short sleep duration plus insomnia symptoms showed results in the same direction of those observed for short sleepers. Therefore, we supposed that most individuals who reported short sleep duration do not have high sleep quality. Third, although the cross-sectional study design did not allow us to infer causality, the literature on sleep and metabolic disorders suggests that sleep problems most likely enhance the probability of developing cardiometabolic outcomes. ${ }^{24}$ Fourth, our plasma measures were obtained based on standard procedures between 7:00 a.m. and 10:00 a.m., but the exact moment of assessment was not recorded. As we expect a diurnal variation for some biological markers, the timing of the blood test could be a potential confounder that was not considered. Fifth, we recognize the relevance of age in analysis of cardiometabolic factors. Nevertheless, our sample size (only 4\% reported long sleep duration) did not allow us to deeply study age effects - for instances, only 41 individuals with high glycated hemoglobin levels reported long sleep duration). Finally, our study sample comprises a particular population (Brazilian civil servants) and the generalization of our findings should be done with caution.

\section{Conclusions}

Our findings contribute to the discussion on the importance of adequate sleep quality and duration and may have important clinical and public health implications, mainly in relation to primary prevention. It is essential to identify modifiable life factors associated with a lower risk for developing noncommunicable diseases, as proposed in the present study. Assessing the associations between sleep problems and cardiometabolic outcomes according to gender contributes to filling the gap in the literature on possible gender differences, highlighted in recent systematic reviews with meta-analyses. The 
investigation of the lipid profile deserves further attention, since studies on this issue are still rare and inconclusive. The study contributes to the recognition that sleeping $>6$ hours to $\leq 8$ hours per night can be treated as presenting the lowest risk for cardiometabolic risk factors. Lastly, the associations of selfreported short sleep duration plus insomnia symptoms with higher likelihoods of obesity, hypertension and high glycated hemoglobin levels reinforces the importance of considering quantitative and qualitative aspects of sleep for the prevention and management of the outcomes.

\section{Abbreviations}

BMl: body mass index

Cl: confidence intervals

ELSA-Brasil: Brazilian Longitudinal Study of Adult Health

FIOCRUZ: Oswaldo Cruz Foundation

HDL-cholesterol: High-density lipoprotein cholesterol

ICC: intraclass correlation coefficient

MET: Metabolic Equivalents

mg/dL: milligrams per deciliter

PA: physical activity

UFBA: Federal University of Bahia

UFES: Federal University of Espirito Santo

UFMG: Federal University of Minas Gerais

UFRGS: Federal University of Rio Grande do Sul

USP: Sao Paulo University

\section{Declarations}

\section{Ethics approval and consent to participate}

The research ethics committees of all six centers approved this study (Federal University of Minas Gerais - UFMG: 186/06; São Paulo University - USP: 669/06; Federal University of Rio Grande do Sul - UFRGS: 194/061; Federal University of Espírito Santo - UFES: 041/06; Federal University of Bahia - UFBA: 027/06; Oswaldo Cruz Foundation - FIOCRUZ: 343/06), and all participants freely signed a declaration of informed consent.

\section{Consent for publication}

Not applicable

\section{Availability of data and materials}

The datasets used and analysed during the current study are available upon request to the Study's Steering Committee, through an appointed representative, Dr. Rosane Harter Griep (rohgriep@gmail.com). The ELSA-Brasil study, while open to any researcher, has a policy of requiring that all proposals of investigations pass through the study's publications committee.

\section{Competing interests}

\section{The authors declare that they have no competing interests}

\section{- Funding}

- The ELSA-Brasil baseline was supported by the Brazilian Ministry of Health (Science and Technology Department) and the Brazilian Ministry of Science and Technology [grants 01060010.00 RS, 01060212.00 BA, 01060300.00 ES, 0106 0278.00 MG, 01060115.00 SP, 01060071.00 RJ]. ASC was supported by a CNPq (Brazilian National Research Council) Postdoc scholarship (150551/2015-0) and FAPEMIG (APQ-01422-17). LR, DC, EMLA, SMB, MIS, and RHG are fellows of the CNPq (Productivity Scholarship).

\section{Authors' contributions}

ASC wrote the first draft of the manuscript. ASC and AAN analyzed the data. All authors made a substantial contribution to the conception and the design of the study. All authors read and approved the final manuscript. 


\section{Acknowledgements}

We thank all ELSA-Brasil participants who agreed to take part in the study

\section{References}

1. Nedeltcheva AV, Scheer FA. Metabolic effects of sleep disruption, links to obesity and diabetes. Curr Opin Endocrinol Diabetes Obes. 2014;21(4):293-8. doi: 10.1097/MED.0000000000000082.

2. Schmid SM, Hallschmid M, Schultes B. The metabolic burden of sleep loss. Lancet Diabetes Endocrinol. 2014;25. doi: 10.1016/S2213-8587(14)70012-9.

3. Meng L, Zheng Y, Hui R. The relationship of sleep duration and insomnia to risk of hypertension incidence: a meta-analysis of prospective cohort studies. Hypertens Res. 2013; 36(11): 985-995. doi: 10.1038/hr.2013.70.

4. Nuyujukian DS, Anton-Culver H, Manson SM, Jiang L. Associations of sleep duration with cardiometabolic outcomes in American Indians and Alaska Natives and other race/ethnicities: results from the BRFSS. Sleep Health.2019; S2352-7218(19)30026-9. doi: 10.1016/j.sleh.2019.02.003.

5. Shan Z, Ma H, Xie M, Yan P, Guo Y, Bao W, Rong Y, Jackson CL, Hu FB, Liu L. Sleep duration and risk of type 2 diabetes: a meta-analysis of prospective studies. Diabetes Care. 2015;38(3):529-37. doi: 10.2337/dc14-2073.

6. Zhou Q, Zhang M, Hu D. Dose-response association between sleep durationand obesity risk: a systematicreview and meta-analysis of prospective cohort studies. Sleep Breath. 2019. doi: 10.1007/s11325-019-01824-4.

7. Cappuccio FP, D'Elia L, Strazzullo P, Miller MA. Quantity and quality of sleep and incidence of type 2 diabetes: a systematic review and meta-analysis. Diabetes Care. 2010;33(2):414-20. doi: 10.2337/dc09-1124.

8. Wang Q, Xi1 B, Liu M, Zhang Y, Fu M. Short sleep duration is associated with hypertension risk among adults: a systematic review and meta-analysis. Hypertension Research 2012;35, 1012-1018. doi: 10.1038/hr.2012.91.

9. Léger D, Beck F, RichardJ, Sauvet F, Faraut B. The Risks of Sleeping “Too Much”. Survey of a National Representative Sample of 24671 Adults (INPES Health Barometer) PLoS One. 2014; 9(9): e106950. doi: 10.1371/journal.pone.0106950.

10. Kruisbrink M, Robertson W, Ji C, Miller MA, Geleijnse JM, Cappuccio FP. Association of sleep duration and quality with blood lipids: a systematic review and meta-analysis of prospective studies. BMJ Open 2017;7:e018585. doi:10.1136/ bmjopen-2017-018585.

11. Itani O, Jike M, Watanabe N, Kaneita Y. Short sleep duration and health outcomes: A systematic review, meta-analysis and meta-regression. Sleep Medicine. 2017;32:246-256. doi: 10.1016/j.sleep.2016.08.006.

12. Jike M, Itani O, Watanabe N, Buysse DJ, Kaneita Y. Long sleep duration and health outcomes: A systematic review, meta-analysis and metaregression. Sleep Medicine Reviews. 2018;39:25-36. doi: 10.1016/j.smrv.2017.06.011.

13. Nuyujukian DS, Beals J, Huang H, Johnson A, Bullock A, Manson SM, Jiang L. Special Diabetes Program for Indians Diabetes Prevention Demonstration Project. Sleep Duration and Diabetes Risk in American Indian and Alaska Native Participants of a Lifestyle Intervention Project. Sleep. 2016;39(11):19191926. Doi: $10.5665 /$ sleep.6216.

14. Anujuo K, Stronks K, Snijder MB, Jean-Louis G, Rutters F, van den Born BJ, Peters RJ, Agyemang C. Relationship between short sleep duration and cardiovascular risk factors in a multi-ethnic cohort - the helius study. Sleep Med. 2015;16(12):1482-8. doi: 10.1016/j.sleep.2015.08.014.

15. Clark AJ, Salo P, Lange T, Jennum P, Virtanen M, Pentti J, Kivimäki M, Rod NH, Vahtera J. Onset of Impaired Sleep and Cardiovascular Disease Risk Factors: A Longitudinal Study. Sleep. 2016 pii: sp-00014-16. doi: 10.5665/sleep.6098.

16. Wu Y, Zhai L, Zhang D. Sleep duration and obesity among adults: a meta-analysis of prospective studies. Sleep Med. 2014;15(12):1456-62. doi: 10.1016/j.sleep.2014.07.018.

17. Cespedes EM, Dudley KA, Sotres-Alvarez D, Zee PC,Daviglus ML, Shah NA, Talavera GA, Gallo LC, Mattei J, Qi Q, Ramos AR, Schneiderman N, Espinozagiacinto RA, Patel SR. Joint associations of insomnia and sleep duration with prevalent diabetes: The Hispanic Community Health Study/Study of Latinos (HCHS/SOL). J Diabetes. 2016; 8(3): 387-397. doi: 10.1111/1753-0407.12308.

18. Li Y, Gao X, Winkelman JW, Cespedes EM, Jackson CL, Walters AS, Schernhammer E, Redline S, Hu FB. Association between sleeping difficulty and type 2 diabetes in women. Diabetologia. 2016;59(4):719-27. doi: 10.1007/s00125-015-3860-9.

19. Chan WS, Levsen MP, McCrae CS. A meta-analysis of associations between obesity and insomnia diagnosis and symptoms. Sleep Med Rev.2018;40:170182. doi: 10.1016/j.smrv.2017.12.004.

20. Grandner MA, Perlis ML. Short sleep duration and insomnia associated with hypertension incidence. Hypertens Res. 2013;36(11):932-3. doi: 10.1038/hr.2013.83.

21. Silva-Costa A, Griep RH, Rotenberg L. Associations of a Short Sleep Duration, Insufficient Sleep, and Insomnia with Self-Rated Health among Nurses. PLoS One 2015;10(5):e0126844. doi: 10.1371/journal.pone.0126844. eCollection 2015.

22. Cai G-H, Theorell-Haglöw J, Janson C, Svartengren M, Elmståhl S, Lind L, Lindberg E. Insomnia symptoms and sleep duration and their combined effects in relation to associations with obesity and central obesity. Sleep Medicine 2018; 46:81-87. doi: 10.1016/j.sleep.2018.03.009.

23. Vgontzas AN, Liao D, Bixler EO, Chrousos GP, Vela-Bueno A. Insomnia with objective short sleep duration is associated with a high risk for hypertension. Sleep 2009; 32(4):491-7.

24. Vgontzas AN, Liao D, Pejovic S, Calhoun S, Karataraki M, Bixler EO. Insomnia with objective short sleep duration is associated with type 2 diabetes: a population-based study. Diabetes Care. 2009a; 32(11): 1980-1985. doi: 10.2337/dc09-0284.

25. Ohayon MM. Epidemiology of insomnia: what we know and what we still need to learn. Sleep Med Rev. 2002;(2):97-111. 
26. Mallampalli MP \& Carter CL. Exploring Sex and Gender Differences in Sleep Health: A Society for Women's Health Research Report. J Womens Health (Larchmt). 2014; 23(7): 553-562. doi: 10.1089/jwh.2014.4816.

27. Prather AA, Epel ES, Cohen BE, Neylan TC, Whooley MA. Gender differences in the prospective associations of self-reported sleep quality with biomarkers of systemic inflammation and coagulation: findings from the Heart and Soul Study. J Psychiatr Res. 2013;47(9). doi: 10.1016/j.jpsychires.2013.05.004.

28. Cappuccio FP, Stranges S, Kandala NB, Miller MA, Taggart FM, Kumari M, Ferrie JE, Shipley MJ, Brunner EJ, Marmot MG. Gender-specific associations of short sleep duration with prevalent and incident hypertension: the Whitehall II Study. Hypertension. 2007;50(4):693-700. Epub 2007 Sep 4. Erratum in: Hypertension. 2007 Nov;50(5):e170. doi: 10.1161/HYPERTENSIONAHA.107.095471.

29. Zhan Y, Chen R, Yu J. Sleep duration and abnormal serum lipids: the China Health and Nutrition Survey. Sleep Med. 2014;15(7):833-9. doi: 10.1016/j.sleep.2014.02.006.

30. Westerlund, Bottai M, Adami H-O, Bellocco R, Nyrén O, Åkerstedt T, Lagerros YT. among Swedish men and women. Sleep Med. 2014;15(10):1196-203. doi: 10.1016/j.sleep.2014.06.012.

31. Ogilvie RP, Bazzano LA, Gustat J, Harville EW, Chen W, Patel SR. Sex and race differences in the association between sleep duration and adiposity: the Bogalusa Heart Study. Sleep Health. 2019;5(1):84-90. doi: 10.1016/j.sleh.2018.10.010.

32. Theorell-Haglöw J, Miller CB, Bartlett DJ, Yee BJ, Openshaw HD, Grunstein RR. Gender differencesin obstructive sleepapnoea, insomnia and restless legs syndrome in adults - What do we know? A clinical update. Sleep Med Rev. 2018; 38:28-38. doi: 10.1016/j.smrv.2017.03.003.

33. Koren D, Dumin M, Gozal D. Role of sleep quality in the metabolic syndrome Diabetes Metab Syndr Obes. 2016; 9: 281-310. doi: 10.2147/DMSO.S95120.

34. Malta DC, Andrade SSCA, Oliveira TP, Moura L, Prado RR, Souza MFM. Probability of premature death for chronic non-communicable diseases, Brazil and Regions, projections to 2025. Revista Brasileira de Epidemiologia 2019;22: e190030. doi.org/10.1590/1980-549720190030.

35. Bensenor IM, Griep RH, Pinto KA, Felisbino-Mendes M, Caetano El, Albuquerque L da S, Schmidt MI. Routines of organization of clinical tests and interviews in the ELSA-Brasil investigation center. Rev Saúde Pública. 2013; 47:37-47.

36. Schmidt MI, Griep RH, Passos VM, Luft VC, Goulart AC, Menezes GM, Molina Mdel C, Vigo A, Nunes MA. Strategies and development of quality assurance and control in the ELSA-Brasil. Rev Saúde Pública. 2013; 47:105-12.

37. Fedeli LG, Vidigal PG, Leite CM, Castilhos CD, Pimentel RA, Maniero V, Mill JG, Lotufo PA, Pereira AC, Bensenor IM. Logistics of collection and transportation of biological samples and the organization of the central laboratory in the ELSA-Brasil. Revista de Saúde Pública 2013;47(Suppl. 2), 63-71. doi.10.1590/s0034-8910.2013047003807.

38. Craig CL, Marshall AL, Sjostrom M, Bauman AE, Both ML, International physical activity questionnaire: 12-country reliability and validity. Med Sci Sports Exerc. 2003; 35(8):1381-95. doi: 10.1249/01.MSS.0000078924.61453.FB.

39. Nunes, MA, Alves, MGM, Chor, D, Schmidt, MI, Duncan, BB. Adaptação transcultural do CIS-R (Clinical Interview Schedule- Revised Version) para o português no Estudo Longitudinal De Saúde Do Adulto (ELSA). Clinical \& Biomedical Research 2011; 31, 487-490. Doi: 10.1097/MED.0000000000000082.

40. Grandner M, Mullington JM, Hashmi SD, Redeker NS, Watson NF, Morgenthaler TI. Sleep Duration and Hypertension: Analysis of > 700,000 Adults by Age and Sex. Journal of Clinical Sleep Medicine: JCSM: Official Publication of the American Academy of Sleep Medicine. 2018;14(6):1031-1039. doi:10.5664/jcsm.7176.

41. Kim CE, Shin S, Lee HW, Lim J, Lee JK, Shin A, Kang D. Association between sleep duration and metabolic syndrome: a cross-sectional study. BMC Public Health. 2018;18(1):720. doi: 10.1186/s12889-018-5557-8.

42. Westerlund A, Bellocco R, Sundström J, Adami HO, Åkerstedt T, Trolle Lagerros Y. Sleep characteristics and cardiovascular events in a large Swedish cohort. Eur J Epidemiol. 2013;28(6):463-73. doi: 10.1007/s10654-013-9802-2.

43. Carter JR, Durocher JJ, Larson RA, Valla JPD, Yang H. Sympathetic neural responses to 24-hour sleep deprivation in humans: sex differences. Am J Physiol Heart Circ Physiol. 2012;302(10): H1991-H1997. doi: 10.1152/ajpheart.01132.2011.

44. Haaramo P, Rahkonen O, Hublin C, Laatikainen T, Lahelma E, Lallukka T. Insomnia symptoms and subsequent cardiovascular medication: a registerlinked follow-up study among middle-aged employees. J Sleep Res. 2014;23(3):281-9. doi: 10.1111/jsr.12116.

45. SongQ, Liu X, Zhou W, Wang X, Wu S. Short-term changes in sleepduration and risk of type 2 diabetes: Kailuan prospective study. Medicine (Baltimore). 2016;95(45):e5363. doi: 10.1097/MD.0000000000005363.

46. García-Perdomo HA, Zapata-Copete J, Rojas-Cerón CA. Sleep durationand risk of all-cause mortality: a systematic review and meta-analysis. Epidemiol Psychiatr Sci.2019;28(5):578-588. doi: 10.1017/S2045796018000379.

\section{Tables}

Table 1: Sleep duration and insomnia symptoms according to sociodemographic and cardiometabolic risk factors by sex. ELSA-Brasil (2013-2014) 


\begin{tabular}{|c|c|c|c|c|c|c|c|}
\hline & \multicolumn{5}{|c|}{ Women $(\mathrm{N}=7491)$} & \multirow{2}{*}{\multicolumn{2}{|c|}{ Self-reported Sle }} \\
\hline & \multicolumn{3}{|c|}{ Self-reported Sleep Duration } & \multicolumn{2}{|c|}{ Insomnia Symptoms } & & \\
\hline & $\begin{array}{c}\leq 6 \mathrm{~h} \\
(\mathrm{n}=3564)\end{array}$ & $\begin{array}{l}7 \mathrm{~h}-8 \mathrm{~h} \\
(\mathrm{n}=3592)\end{array}$ & $\begin{array}{c}>8 \mathrm{~h} \\
(\mathrm{n}=335)\end{array}$ & $\begin{array}{c}\text { No } \\
(\mathrm{n}=5406)\end{array}$ & $\begin{array}{c}\text { Yes } \\
(\mathrm{n}=2085)\end{array}$ & $\begin{array}{c}\leq 6 \mathrm{~h} \\
(\mathrm{n}=3063)\end{array}$ & $\begin{array}{c}7 \mathrm{~h}-8 \text { h } \\
(\mathrm{n}=2968)\end{array}$ \\
\hline tge (year)* & $55.7(8.8)$ & $\begin{array}{l}55.4 \\
(8.6)\end{array}$ & $\begin{array}{l}57.8 \\
(9.6)\end{array}$ & $55.6(8.7)$ & $56.1(8.8)$ & $55.5(8.9)$ & $55.8(9.5)$ \\
\hline \multicolumn{8}{|l|}{ 'ducation - } \\
\hline 'undamental & $332(9.3)$ & 237 (6.6) & $\begin{array}{c}50 \\
(14.9)\end{array}$ & $358(6.6)$ & $261(12.5)$ & $\begin{array}{c}473 \\
(15.4)\end{array}$ & $385(13.0)$ \\
\hline Iigh school & 1235 (34.6) & $\begin{array}{l}1041 \\
(29.0)\end{array}$ & $\begin{array}{c}134 \\
(40.0)\end{array}$ & $1675(31.0)$ & $735(35.3)$ & $\begin{array}{c}967 \\
(31.6)\end{array}$ & $859(28.9)$ \\
\hline 'ollege & $1997(56.1)$ & $\begin{array}{l}2314 \\
(64.4)\end{array}$ & $\begin{array}{c}151 \\
(45.1)\end{array}$ & $3373(62.4)$ & $1089(52.2)$ & $\begin{array}{l}1623 \\
(53.0)\end{array}$ & $1724(58.1)$ \\
\hline \multicolumn{8}{|c|}{ 'hysical activity-n(\%) } \\
\hline Jo & $2848(79.9)$ & $\begin{array}{l}2694 \\
(75.0)\end{array}$ & $\begin{array}{c}278 \\
(83.0)\end{array}$ & $4116(76.1)$ & $1704(81.7)$ & $\begin{array}{l}2199 \\
(71.8)\end{array}$ & $2008(67.6)$ \\
\hline Ioderate & $514(14.2)$ & $\begin{array}{c}675 \\
(18.8)\end{array}$ & $\begin{array}{c}44 \\
(13.1)\end{array}$ & $946(17.5)$ & $287(13.8)$ & $541(17.7)$ & $632(21.3)$ \\
\hline Iigh & $202(5.7)$ & $223(6.2)$ & $13(3.9)$ & $344(6.4)$ & $94(4.5)$ & $\begin{array}{c}323 \\
(10.5)\end{array}$ & $328(11.1)$ \\
\hline \multicolumn{8}{|l|}{$\mathrm{MI}-\mathrm{n}(\%)$} \\
\hline Jon obese & $2436(68.4)$ & $\begin{array}{l}2677 \\
(74.5)\end{array}$ & $\begin{array}{c}208 \\
(62.1)\end{array}$ & 3927 (72.6) & $1394(66.9)$ & $\begin{array}{l}2343 \\
(76.5)\end{array}$ & $2293(77.3)$ \\
\hline bese & $1128(31.6)$ & $\begin{array}{c}915 \\
(25.5)\end{array}$ & $\begin{array}{c}127 \\
(37.9)\end{array}$ & 1479 (27.6) & $691(33.1)$ & $\begin{array}{c}720 \\
(23.5)\end{array}$ & 675 (22.7) \\
\hline \multicolumn{8}{|l|}{.ow HDL - } \\
\hline Jo & $2556(71.7)$ & $\begin{array}{l}2694 \\
(75.0)\end{array}$ & $\begin{array}{c}233 \\
(69.6)\end{array}$ & 4017 (74.3) & $1466(70.3)$ & $\begin{array}{l}2388 \\
(77.9)\end{array}$ & $2324(78.3)$ \\
\hline es & $1008(28.3)$ & $\begin{array}{c}898 \\
(25.0)\end{array}$ & $\begin{array}{c}102 \\
(30.4)\end{array}$ & $1389(25.7)$ & 619 (29.7) & $\begin{array}{c}675 \\
(22.1)\end{array}$ & $644(21.7)$ \\
\hline \multicolumn{8}{|c|}{ Aypertriglyceridemia n(\%) } \\
\hline Jo & $2787(78.2)$ & $\begin{array}{l}2868 \\
(79.8)\end{array}$ & $\begin{array}{c}254 \\
(75.8)\end{array}$ & $4325(80.0)$ & $1584(76.0)$ & $1996(65.2)$ & $1922(6$ \\
\hline es & 777 (21.8) & $\begin{array}{c}724 \\
(20.2)\end{array}$ & $\begin{array}{c}81 \\
(24.2)\end{array}$ & $1081(20.0)$ & $501(24.0)$ & $1067(34.8)$ & $1046(3$ \\
\hline \multicolumn{8}{|c|}{ Iigh glycated hemoglobin - n(\%) } \\
\hline Jo & $3278(91.9)$ & $\begin{array}{l}3375 \\
(93.9)\end{array}$ & $\begin{array}{c}294 \\
(87.8)\end{array}$ & $5066(93.7)$ & $1881(90.2)$ & 2765 (90.3) & $2676(9$ \\
\hline es & $286(8.1)$ & $217(6.1)$ & $\begin{array}{c}41 \\
(12.2)\end{array}$ & $340(6.3)$ & $204(9.8)$ & $298(9.7)$ & $292(9$ \\
\hline Iypertension & & & & & & & \\
\hline $\mathrm{n}(\%)$ & & & & & & & \\
\hline Jo & $2151(60.3)$ & 2381 & 169 & $3513(65.0)$ & $1188(57.0)$ & $1682(54.9)$ & $1652(55.7)$ \\
\hline
\end{tabular}


lepressive symptoms -

(33.7) (49.6)

(\%)

Jo 2782 (78.1)3101 (86.3)283 (84.5)4800 (88.8)1366 (65.5) 2714 (88.6)2757 (92.9)180 (90.0)4702 (93.5)949 (79.1)

es $782(21.9) \quad 491(13.7) \quad 52(15.5) \quad 606(11.2) \quad 719(34.5) \quad 211(11.4) \quad 349(7.1) \quad 20(10.0) \quad 329(6.5) \quad 251(20.9)$

Table 2: Association (odds ratio [95\%CI]) between sleep patterns and cardiometabolic risk factors among women. ELSA-Brasil, 2013-2014 


\begin{tabular}{|c|c|c|c|c|c|}
\hline \multicolumn{6}{|c|}{ Obesity } \\
\hline & Model 1 & Model 2 & Model 3 & Model 4 & Model 5 \\
\hline \multicolumn{6}{|c|}{ Self-reported sleep duration } \\
\hline$>6-\leq 8$ hours & \multicolumn{5}{|c|}{ Reference category } \\
\hline$<=6$ hours & $1.35(1.22 ; 1.50)$ & $1.29(1.16 ; 1.43)$ & $1.25(1.13 ; 1.39)$ & $1.23(1.10 ; 1.37)$ & $1.22(1.09 ; 1.36)$ \\
\hline$>8$ hours & $1.79(1.41 ; 2.25)$ & $1.59(1.25 ; 2.01)$ & $1.55(1.22 ; 1.97)$ & $1.56(1.23 ; 1.97)$ & $1.56(1.23 ; 1.98)$ \\
\hline \multicolumn{6}{|c|}{ Insomnia symptoms } \\
\hline No & \multicolumn{5}{|c|}{ Reference category } \\
\hline Yes & $1.32(1.18 ; 1.47$ & $1.23(1.10 ; 1.38)$ & $1.15(1.02 ; 1.29)$ & $1.09(0.97 ; 1.23)$ & $1.09(0.96 ; 1.23)$ \\
\hline \multicolumn{6}{|c|}{ Short sleep + insomnia } \\
\hline \multicolumn{3}{|c|}{ No (>6 - 8 hours and absence of insomnia) } & \multicolumn{3}{|c|}{ Reference category } \\
\hline \multirow[t]{2}{*}{ Yes } & $1.58(1.38 ; 1.81)$ & $1.43(1.25 ; 1.65)$ & $1.32(1.14 ; 1.53)$ & - & $1.31(1.13 ; 1.51)$ \\
\hline & \multicolumn{5}{|l|}{ Hypertension } \\
\hline \multicolumn{6}{|c|}{ Self-reported sleep duration } \\
\hline$>6-\leq 8$ hours & \multicolumn{5}{|c|}{ Reference category } \\
\hline$<=6$ hours & $1.29(1.17 ; 1.42)$ & $1.29(1.17 ; 1.43)$ & $1.19(1.08 ; 1.33)$ & $1.15(1.03 ; 1.28)$ & $1,15(1.03 ; 1.28)$ \\
\hline$>8$ hours & $1.93(1.54 ; 2.42)$ & $1.73(1.36 ; 2.19)$ & $1.52(1.19 ; 1.93)$ & $1.53(1.20 ; 1.94)$ & $1.53(1.20 ; 1.95)$ \\
\hline \multicolumn{6}{|c|}{ Insomnia symptoms } \\
\hline No & \multicolumn{5}{|c|}{ Reference category } \\
\hline Yes & $1.40(1.26 ; 1.55)$ & $1.39(1.24 ; 1.54)$ & $1.22(1.09 ; 1.37)$ & $1.19(1.05 ; 1.33)$ & $1.18(1.05 ; 1.33)$ \\
\hline \multicolumn{6}{|c|}{ Short sleep + insomnia } \\
\hline \multicolumn{6}{|c|}{ No ( $>6-8$ hours and absence of insomnia) } \\
\hline Yes & $1.62(1.43 ; 1.84)$ & $1.46(1.28 ; 1.67)$ & $1.37(1.19 ; 1.58)$ & - & $1.33(1.15 ; 1.53)$ \\
\hline \multicolumn{6}{|c|}{ High glycated hemoglobin } \\
\hline \multicolumn{6}{|c|}{ Self-reported sleep duration } \\
\hline$>6-\leq 8$ hours & Reference categ & & & & \\
\hline$<=6$ hours & $1.36(1.13 ; 1.63)$ & $1.35(1.12 ; 1.62)$ & $1.20(1.00 ; 1.45)$ & $1.12(0.92 ; 1.36)$ & $1.11(0.91 ; 1.36)$ \\
\hline$>8$ hours & $2.17(1.50 ; 3.06)$ & $1.90(1.31 ; 2.70)$ & $1.62(1.11 ; 2.32)$ & $1.64(1.13 ; 2.34)$ & $1.65(1.13 ; 2.23)$ \\
\hline Insomnia symptc & & & & & \\
\hline No & Reference categ & & & & \\
\hline Yes & $1.62(1.35 ; 1.94)$ & $1.59(1.31 ; 1.89)$ & $1.32(1.09 ; 1.60)$ & $1.31(1.06 ; 1.61)$ & $1.30(1.06 ; 1.60)$ \\
\hline Short sleep + in & mnia & & & & \\
\hline No $(>6-8$ hours & nd absence of insc & aia) & ategory & & \\
\hline Yes & $1.91(1.52 ; 2.39)$ & $1.60(1.27 ; 2.01)$ & $1.48(1.17 ; 1.88)$ & - & $1.43(1.12 ; 1.82)$ \\
\hline & & Hyperts & & & \\
\hline Self-reported sle & duration & & & & \\
\hline$>6-\leq 8$ hours & Reference categ & & & & \\
\hline$<=6$ hours & $1.10(0.99 ; 1.24)$ & $1.10(0.98 ; 1.23)$ & $1.05(0.93 ; 1.17)$ & $1.04(0.89 ; 1.13)$ & $0.99(0.88 ; 1.12)$ \\
\hline$>8$ hours & $1.26(0.96 ; 1.64)$ & $1.20(0.91 ; 1.55)$ & $1.11(0.84 ; 1.44)$ & $1.11(0.85 ; 1.44)$ & $1.12(0.86 ; 1.46)$ \\
\hline Insomnia symptc & & & & & \\
\hline No & Reference categ & & & & \\
\hline Yes & $1.27(1.12 ; 1.43)$ & $1.25(1.11 ; 1.41)$ & $1.17(1.03 ; 1.33)$ & $1.17(1.03 ; 1.34)$ & $1.16(1.01 ; 1.32)$ \\
\hline Short sleep + in & mnia & & & & \\
\hline No ( $>6-8$ hours & nd absence of insc & nia) & erence category & & \\
\hline Yes & $1.31(1.13 ; 1.52)$ & $1.23(1.06 ; 1.43)$ & $1.18(1.01 ; 1.38)$ & - & $1.12(0.95 ; 1.31)$ \\
\hline & & Low HD & & & \\
\hline Self-reported sle & duration & & & & \\
\hline$>6-\leq 8$ hours & Reference categ & & & & \\
\hline$<=6$ hours & $1.18(1.06 ; 1.31)$ & $1.191 .07 ; 1.32)$ & $1.13(1.01 ; 1.25)$ & $1.10(0.99 ; 1.23)$ & $1.10(0.98 ; 1.23)$ \\
\hline$>8$ hours & $1.31(1.03 ; 1.67)$ & $1.36(1.06 ; 1.74)$ & $1.24(0.97 ; 1.59)$ & $1.25(0.97 ; 1.59)$ & $1.25(0.97 ; 1.59)$ \\
\hline Insomnia symptc & & & & & \\
\hline No & Reference categ & & & & \\
\hline Yes & $1.22(1.09 ; 1.36)$ & $1.23(1.10 ; 1.38)$ & $1.13(1.00 ; 1.27)$ & $1.10(0.97 ; 1.25)$ & $1.10(0.96 ; 1.25)$ \\
\hline Short sleep + in & mnia & & & & \\
\hline No $(>6-8$ hours & nd absence of insc & nia) & ce category & & \\
\hline Yes & $1.33(1.16 ; 1.53)$ & $1.27(1.10 ; 1.46)$ & $1.21(1.04 ; 1.39)$ & - & $1.19(1.03 ; 1.38)$ \\
\hline
\end{tabular}


Table 3: Association (odds ratio [95\%CI]) between sleep patterns and cardiometabolic risk factors among men. ELSA-Brasil, 2013-2014

\begin{tabular}{|c|c|c|c|c|}
\hline & \multicolumn{4}{|c|}{ Obesity } \\
\hline & Model 1 & Model 2 & Model 3 & Model 4 \\
\hline \multicolumn{5}{|c|}{ Self-reported sleep duration } \\
\hline$>6-\leq 8$ hours & \multicolumn{4}{|c|}{ Reference category } \\
\hline$<=6$ hours & $1.04(0.93 ; 1.18)$ & $1.03(0.91 ; 1.16)$ & $1.02(0.90 ; 1.15)$ & $1.02(0.90 ; 1.16)$ \\
\hline$>8$ hours & $1.26(0.90 ; 1.73)$ & $1.22(0.87 ; 1.62)$ & $1.20(0.86 ; 1.65)$ & $1.06(0.86 ; 1.65)$ \\
\hline \multicolumn{5}{|c|}{ Insomnia symptoms } \\
\hline No & \multicolumn{4}{|c|}{ Reference category } \\
\hline Yes & $1.01(0.87 ; 1.17)$ & $1.00(0.86 ; 1.16)$ & $0.99(0.85 ; 1.15)$ & $0.99(0.84 ; 1.16)$ \\
\hline \multicolumn{5}{|c|}{ Short sleep + insomnia } \\
\hline \multicolumn{2}{|c|}{ No (>6 - 8 hours and absence of insomnia) } & \multicolumn{3}{|l|}{ Reference category } \\
\hline \multirow[t]{2}{*}{ Yes } & $1.07(0.89 ; 1.27)$ & $1.04(0.87 ; 1.27)$ & $1.02(0.85 ; 1.23)$ & - \\
\hline & \multicolumn{4}{|l|}{ Hypertension } \\
\hline \multicolumn{4}{|c|}{ Self-reported sleep duration } & Reference category \\
\hline$<=6$ hours & $1.03(0.93 ; 1.14)$ & $1.04(0.94 ; 1.16)$ & $1.03(0.93 ; 1.15)$ & $1.02(0.91 ; 1.14)$ \\
\hline$>8$ hours & $1.19(0.89 ; 1.59)$ & $1.06(0.78 ; 1.44)$ & $1.06(0.78 ; 1.43)$ & $1.06(0.78 ; 1.43)$ \\
\hline \multicolumn{5}{|c|}{ Insomnia symptoms } \\
\hline No & \multicolumn{4}{|c|}{ Reference category } \\
\hline Yes & $1.18(1.04 ; 1.34)$ & $1.09(0.96 ; 1.25)$ & $1.07(0.94 ; 1.23)$ & $1.07(0.93 ; 1.23)$ \\
\hline \multicolumn{5}{|c|}{ Short sleep + insomnia } \\
\hline \multicolumn{2}{|c|}{ No ( $>6$ - 8 hours and absence of insomnia) } & \multicolumn{3}{|l|}{ Reference category } \\
\hline Yes & $1.21(1.04 ; 1.41)$ & $1.13(0.97 ; 1.33)$ & $1.11(0.94-1.30)$ & - \\
\hline & & High glycated & hemoglobin & \\
\hline Self-reported sl & & & & \\
\hline$>6-\leq 8$ hours & Reference categ & & & \\
\hline$<=6$ hours & $0.99(0.83 ; 1.17)$ & $0.97(0.82 ; 1.15)$ & $0.95(0.79 ; 1.12)$ & $0.93(0.78 ; 1.11)$ \\
\hline$>8$ hours & $1.07(0.65 ; 1.67)$ & $0.88(0.53 ; 1.39)$ & $0.87(0.53 ; 1.37)$ & $0.87(0.53 ; 1.37)$ \\
\hline Insomnia sympt & & & & \\
\hline No & Reference categ & & & \\
\hline Yes & $1.25(1.02 ; 1.53)$ & $1.11(0.90 ; 1.36)$ & $1.04(0.84 ; 1.28)$ & $1.06(0.85 ; 1.32)$ \\
\hline Short sleep + in & & & & \\
\hline No ( $>6$ - 8 hours & mnia) & Reference categ & & \\
\hline Yes & $1.19(0.93 ; 1.51)$ & $1.05(0.82 ; 1.34)$ & $0.98(0.75 ; 1.25)$ & - \\
\hline & & Hypertrigly & eridemia & \\
\hline Self-reported sl & & & & \\
\hline$>6-\leq 8$ hours & Reference categ & & & \\
\hline$<=6$ hours & $0.98(0.88 ; 1.09)$ & $0.97(0.87 ; 1.08)$ & $0.96(0.86 ; 1.07)$ & $0.93(0.84 ; 1.04)$ \\
\hline$>8$ hours & $1.65(1.24 ; 2.19)$ & $1.60(1.20 ; 2.14)$ & $1.59(1.19 ; 2.13)$ & $1.60(1.20 ; 2.14)$ \\
\hline Insomnia sympt & & & & \\
\hline No & Reference categ & & & \\
\hline Yes & $0.98(0.84 ; 1.14)$ & $1.13(0.99 ; 1.29)$ & $1.13(0.99 ; 1.29)$ & $1.17(1.02 ; 1.35)$ \\
\hline Short sleep + in & & & & \\
\hline No $(>6-8$ hours & mnia) & Reference categc & & \\
\hline Yes & $1.14(0.97 ; 1.33)$ & $1.12(0.96 ; 1.31)$ & $1.11(0.94 ; 1.30)$ & - \\
\hline & & Low HDL-ch & olesterol & \\
\hline Self-reported sl & & & & \\
\hline$>6-\leq 8$ hours & Reference categ & & & \\
\hline$<=6$ hours & $1.02(0.90 ; 1.15)$ & $1.01(0.89 ; 1.14)$ & $0.99(0.88 ; 1.12)$ & $1.01(0.89 ; 1.14)$ \\
\hline$>8$ hours & $0.98(0.69 ; 1.38)$ & $0.95(0.66 ; 1.33)$ & $0.93(0.65 ; 1.31)$ & $0.93(0.65 ; 1.31)$ \\
\hline Insomnia sympt & & & & \\
\hline No & Reference categ & & & \\
\hline Yes & $0.98(0.84 ; 1.14)$ & $0.96(0.83 ; 1.12)$ & $0.93(0.80 ; 1.09)$ & $0.93(0.79 ; 1.09)$ \\
\hline Short sleep + in & & & & \\
\hline No $(>6-8$ hours & mnia) & Reference categ & & \\
\hline Yes & $1.01(0.84 ; 1.21)$ & $0.98(0.82 ; 1.18)$ & $0.94(0.78 ; 1.13)$ & - \\
\hline
\end{tabular}



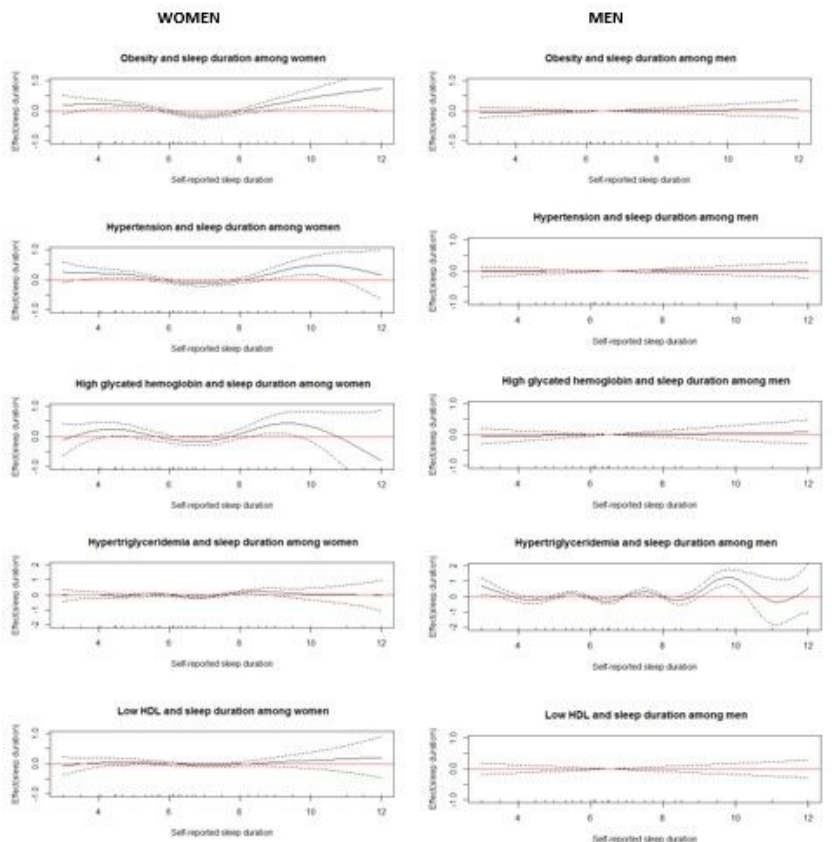

Figure 1

Generalized Additive Models (adjusted for all potential confounders) were used to evaluate the curve shape for the relationship between self-reported sleep duration and the outcomes. The solid black line represents the regression line and the dotted lines represent the $95 \%$ confidence interval. The horizontal red line indicates that there is no association. 